RESPIRATORY MUSCLES

\title{
Effect of salmeterol on respiratory muscle activity during exercise in poorly reversible COPD
}

\author{
W D C Man, N Musiffa, D Nikoletou, S Kaul, N Hart, G F Rafferty, N Donaldson, M I Polkey, \\ J Moxham
}

Thorax 2004;59:471-476. doi: 10.1136/thx.2003.019620

See end of article for authors' affiliations

.....................

Correspondence to: Dr W D C Man, Respiratory Muscle Laboratory, Guy's, King's and St Thomas' School of Medicine, King's College Hospital, Bessemer Road, London SE5 9PJ, UK; william.man@kcl.ac.uk

Received 2December 2003 Accepted

25 February 2004
Background: Some patients with irreversible chronic obstructive pulmonary disease (COPD) experience subjective benefit from long acting bronchodilators without change in forced expiratory volume in 1 second $\left(\mathrm{FEV}_{1}\right)$. Dynamic hyperinflation is an important determinant of exercise induced dyspnoea in COPD. We hypothesised that long acting bronchodilators improve symptoms by reducing dynamic hyperinflation and work of breathing, as measured by respiratory muscle pressure-time products.

Methods: Sixteen patients with "irreversible" COPD $(<10 \%$ improvement in FEV 1 following a bronchodilator challenge; mean $\mathrm{FEV}_{1} 31.1 \%$ predicted) were recruited into a randomised, double blind, placebo controlled, crossover study of salmeterol (50 $\mathrm{gg}$ twice a day). Treatment periods were of 2 weeks duration with a 2 week washout period. Primary outcome measures were end exercise isotime transdiaphragmatic pressure-time product and dynamic hyperinflation as measured by inspiratory capacity.

Results: Salmeterol significantly reduced the transdiaphragmatic pressure-time product $(294.5 \mathrm{v}$ $348.6 \mathrm{~cm} \mathrm{H} 2 \mathrm{O} / \mathrm{s} / \mathrm{min} ; \mathrm{p}=0.03$ ), dynamic hyperinflation (0.22 $v 0.33$ litres; $p=0.002$ ), and Borg scores during endurance treadmill walk (3.78 $v 4.62 ; p=0.02)$. There was no significant change in exercise endurance time. Improvements in isotime Borg score were significantly correlated to changes in tidal volume/oesophageal pressure swings, end expiratory lung volume, and inspiratory capacity, but not pressure-time products.

Conclusions: Despite apparent "non-reversibility" in spirometric parameters, long acting bronchodilators can cause both symptomatic and physiological improvement during exercise in severe COPD.
B ronchodilators are the mainstay of treatment in chronic obstructive pulmonary disease (COPD). ${ }^{1}$ Inhaled long acting $\beta_{2}$ agonists improve control of symptoms and health status ${ }^{23}$ and reduce the need for rescue medications and the frequency of exacerbations. ${ }^{4}$

Traditionally, improvement in forced expiratory volume in 1 second $\left(\mathrm{FEV}_{1}\right)$ has been used to measure the bronchodilator response in COPD. However, some COPD patients with little or no change in $\mathrm{FEV}_{1}$ experience improvement in symptoms with long acting $\beta_{2}$ agonists. The response of an individual patient to a bronchodilator challenge on a single occasion does not predict whether or not the patient will benefit subsequently from that agent. ${ }^{5}$ Furthermore, some investigators have found no correlation between improvements in exertional dyspnoea and changes in $\mathrm{FEV}_{1} .{ }^{6}$ These findings could be explained if other physiological mechanisms operated in the absence of a change in $\mathrm{FEV}_{1}$.

Dynamic hyperinflation is an important determinant of exertional dyspnoea. ${ }^{6}$ During exercise, increased airways resistance and expiratory airflow limitation increase end expiratory lung volume (EELV). The resulting intrinsic positive end expiratory pressure (PEEPi) acts as an inspiratory threshold load that increases the work of breathing as well as placing the diaphragm and other inspiratory muscles at a mechanical disadvantage. It is conceivable that reducing airways resistance and subsequent dynamic hyperinflation with bronchodilators would unload the respiratory muscles by reducing the inspiratory load and work of breathing.

Work of breathing is a complex variable to measure, and many investigators use the pressure-time product (PTP) as an indicator of respiratory muscle activity. The PTP correlates well with measurements of oxygen consumption of contract- ing respiratory muscles, ${ }^{8}$ and has been used to examine respiratory muscle activity in normal subjects, ${ }^{9}$ in patients with $\mathrm{COPD}^{10}$ and in weaning patients in the intensive care setting. ${ }^{11}$

We therefore hypothesised that, in a group of patients with COPD who show no improvement in $\mathrm{FEV}_{1}$ with bronchodilator therapy, the improvement in symptoms would be due to a reduction in dynamic hyperinflation and therefore a reduction in the work of the respiratory muscles. To test our hypothesis we performed a randomised, placebo controlled, double blind, crossover study to examine the effects of a long acting bronchodilator, salmeterol, on dynamic hyperinflation and respiratory muscle PTP during exercise.

\section{METHODS}

\section{Patient selection}

Twenty patients with COPD were recruited. All had been cigarette smokers of at least 20 pack years and had a clinical course consistent with the British Thoracic Society criteria for diagnosis. ${ }^{12}$ Other inclusion criteria included less than $10 \%$ and $200 \mathrm{ml}$ improvement in $\mathrm{FEV}_{1}$ following a bronchodilator challenge (20 minutes after $200 \mu \mathrm{g}$ salbutamol via a metered

Abbreviations: $\mathrm{DH}$, dynamic hyperinflation; EELV, end expiratory lung volume; EILV, end inspiratory lung volume $\mathrm{FEV}_{1}$, forced expiratory volume in 1 second; IC, inspiratory capacity; IRV, inspiratory reserve volume; ISW, incremental shuttle walk; $\mathrm{PaO}_{2}, \mathrm{PaCO}_{2}$, arterial oxygen and carbon dioxide tensions; PEEPi, intrinsic positive end expiratory pressure; PImax, maximum inspiratory pressure; Pdi, diaphragmatic pressure; Pga, gastric pressure; Poes, oesophageal pressure; PTP, pressure-time product; RV, residual volume; TLCO, carbon monoxide transfer factor; TLC, total lung capacity; VC, vital capacity; $\mathrm{VO}_{2}$ peak, peak oxygen consumption 
dosed inhaler) and no infective exacerbation in the preceding 6 weeks. The King's College Hospital research ethics committee approved the protocol and all participants gave informed written consent.

\section{Study design/medications}

The study was of a randomised, double blind, placebo controlled, crossover design with a run in period of 2 weeks and consisted of five outpatient visits. On visit 1 , subjects performed full baseline lung function tests, including formal bronchodilator reversibility, and incremental shuttle walks to determine predicted peak oxygen consumption $\left(\mathrm{VO}_{2}\right.$ peak). Patients stable on inhaled corticosteroids remained on this medication throughout the trial. All other medications apart from rescue short acting $\beta_{2}$ agonists were stopped at the start of the run in period between visits 1 and 2; patients with a symptomatic exacerbation or spirometric deterioration during the run in period were withdrawn from the study and restarted on their usual medication. During both visits 1 and 2 subjects were familiarised with treadmill walking and the performance of inspiratory capacity manoeuvres at rest and during treadmill walking. At the end of visit 2 subjects were randomised to receive either placebo followed by salmeterol $(50 \mu \mathrm{g})$ twice a day or salmeterol followed by placebo using a computer generated randomisation schedule, the Patient Allocation for Clinical Trials. Treatments were given via matching metered dose inhalers and spacer for 2 weeks with a 2 week washout period between visits 3 and 4 . Posttreatment measurements were made on visits 3 and 5 at the same time of day for each patient. The study medication (salmeterol or placebo) was taken 2 hours before lung function tests and 3 hours before the exercise tests. In terms of "rescue therapy", patients were allowed short acting $\beta_{2}$ agonists during treatment periods, but these were stopped for at least 8 hours before measurements were made.

\section{Exercise tests}

On visits 3 and 5 an endurance treadmill test was performed, as well as spirometry and total lung capacity (TLC) obtained from body plethysmography. The chosen walking speed was that producing $80 \%$ of predicted $\mathrm{VO}_{2}$ peak, with predicted $\mathrm{VO}_{2}$ peak calculated using a regression equation from the maximum exercise distance achieved during the baseline incremental shuttle walk at visit $1 .{ }^{13}$ Patients were given standardised encouragement at 1 minute intervals during the walking test to continue exercising until exhaustion. During the treadmill walk, flow was measured by a pneumotachograph (F300L, GM Instruments Ltd, Kilwinning, UK) and tidal volume integrated from inspiratory flow. Operational lung volumes were evaluated from measurements of inspiratory capacity (IC) ${ }^{14}$ at rest, every minute during exercise, and at end exercise, while dyspnoea was assessed by recording modified Borg scores ${ }^{15}$ at 1 minute intervals. Each patient was given a few breaths warning before each IC manoeuvre, and then verbally encouraged to make a maximal effort before relaxing. Oesophageal and transdiaphragmatic pressure swings during the IC manoeuvres were observed to ensure uniformity of maximal effort. As TLC is assumed not to change during exercise, ${ }^{16}{ }^{17}$ the following operational lung volumes were calculated:

- end expiratory lung volume $($ EELV) $=$ TLC - IC;

- inspiratory reserve volume (IRV) = IC - tidal volume;

- end inspiratory lung volume (EILV) = TLC - IRV.

\section{Respiratory muscle activity}

Oesophageal pressure (Poes) and gastric pressure (Pga) were measured at rest and during exercise using conventionally placed balloon catheters attached to differential pressure transducers (MP45, Validyne, CA, USA). Transdiaphragmatic pressures (Pdi) were obtained online by subtraction of Poes from Pga. All signals were digitised using a NB-MIO-16 analogue-digital converter (National Instruments, Texas, USA) and acquired on a Macintosh PowerMac 7600 computer running LabVIEW-4 software (National Instruments, Texas, USA). The sampling rate was $100 \mathrm{~Hz}$.

Inspiratory muscle strength was assessed using volitional tests: maximum inspiratory mouth pressure (PImax), and oesophageal and transdiaphragmatic pressures during a maximal sniff (sniff Poes, sniff Pdi) as previously described. ${ }^{18}$ The pressure-time products (PTPs) of Pdi, Poes, and Pga (PTPdi, PTPoes, and PTPga) were obtained by multiplying the area subtended by the pressure trace by the respiratory frequency and had units of $\mathrm{cm}_{2} \mathrm{O} / \mathrm{s} / \mathrm{min}$. PTPdi and PTPoes were calculated during inspiration of each breath, while PTPga was calculated during expiration (the duty cycle being defined by the appropriate zero points of flow). For PTPdi and PTPga, the baseline was determined for each breath as the level observed at the start of inspiration and expiration, respectively. As the baseline for PTPoes may be overestimated as a result of abdominal muscle action, ${ }^{19}$ this was determined for each breath by the method validated by Appendini and colleagues. $^{20}$ This involved subtracting the decrease in Pga from the fall in Poes during the interval between the onset of inspiratory effort and the point of zero flow. The mean PTPs presented in the results were calculated from the PTP values obtained after analysing each individual breath in the preceding 30 seconds before isotime.

To estimate the mechanoventilatory association of the respiratory system (a crude estimate of compliance), tidal volume divided by the difference in oesophageal pressure during points of zero flow ( $\Delta \mathrm{VT} / \Delta$ Poes) was calculated breath by breath.

\section{Data and statistical analysis}

Based on previous PTP data during exercise in COPD patients, ${ }^{10}$ a minimum of 15 patients were required to show a reduction of $50 \mathrm{~cm} \mathrm{H}_{2} \mathrm{O}$ in PTPdi with $80 \%$ power. Primary outcome measures were PTPdi and IC at end exercise isotime (ISO). ISO was the highest equivalent exercise endurance time between the two treadmill walks.

Statistical analysis was performed using SPSS 11.1 for Windows. Patients were divided into two groups according to the treatment sequence. Since this was a crossover trial, treatment effect was assessed after both period effect and treatment-period interactions had been assessed using two sample $t$ tests. In the absence of period effect and treatmentperiod interaction, treatment effect was assessed with paired $t$ tests or non-parametric equivalent depending on whether the groups were normally distributed. If a significant treatment-period interaction was found at the $10 \%$ significance level, the treatment effect was assessed with a two sample $t$ test, disregarding data from the second period. Univariate and multivariate regression analysis was used to compare the effect of salmeterol on isotime Borg score change with isotime lung volumes, dynamic hyperinflation, respiratory muscle activity and mechanics, as well as changes in static lung function.

\section{RESULTS}

Sixteen of the 20 patients completed the study; two were withdrawn because of worsening of symptoms during the run in period and two developed infective exacerbations during the treatment periods (one while on placebo and the other on salmeterol). Ten of the 16 patients received salmeterol as the first intervention. Baseline characteristics of the remaining participants are shown in table 1 . Of note, 


\begin{tabular}{|c|c|}
\hline & Mean (SD) \\
\hline $\operatorname{Sex}(M: F)$ & 10:6 \\
\hline Age (years) & $68.1(7.6)$ \\
\hline Smoking history (pack years) & $35.3(13.8)$ \\
\hline Pre- $\mathrm{BD} \mathrm{FEV}_{1}$ (I) & $0.76(0.17)$ \\
\hline Post-BD FEV (I) & $0.77(0.15)$ \\
\hline $\mathrm{FEV}_{1}(\%$ pred) & $31.1(3.9)$ \\
\hline $\mathrm{FEV}_{1} / \mathrm{VC}(\%)$ & $32.2(8.1)$ \\
\hline TLCO (\% pred) & $38.8(14.8)$ \\
\hline TLC (\% pred) & $115(14.5)$ \\
\hline RV (\% pred) & $139(14.8)$ \\
\hline $\mathrm{PaO}_{2}(\mathrm{kPa})$ & $8.7(0.9)$ \\
\hline $\mathrm{PaCO}_{2}(\mathrm{kPa})$ & $5.3(0.6)$ \\
\hline ISW distance $(\mathrm{m})$ & $275(75)$ \\
\hline \multicolumn{2}{|c|}{$\begin{array}{l}\mathrm{BD}=\text { bronchodilator trial; } \mathrm{FEV}_{1}=\text { forced expiratory volume in } \\
1 \text { second; } \mathrm{VC}=\text { vital capacity; } \mathrm{TLCO}=\text { carbon monoxide } \\
\text { transfer factor; } \mathrm{TLC}=\text { total lung capacity; } \mathrm{RV}=\text { residual } \\
\text { volume; } \mathrm{PaO}_{2}, \mathrm{PacO}_{2}=\text { arterial oxygen and carbon dioxide } \\
\text { tensions; } \mathrm{ISW}=\text { incremental shuttle walk. }\end{array}$} \\
\hline
\end{tabular}

mean change in $\mathrm{FEV}_{1}$ was only $10 \mathrm{ml}$ following a bronchodilator reversibility test.

\section{Lung function and respiratory muscle strength}

Salmeterol had no significant effect on spirometric measurements or TLC, although resting hyperinflation appeared to be reduced as evidenced by a reduced RV:TLC ratio and an increased IC (tables 2 and 3). There were no changes in inspiratory muscle strength (table 2 ).

\section{Endurance treadmill exercise}

Salmeterol did not have a significant effect on tidal volume, respiratory frequency, minute ventilation, inspiratory/expiratory time, inspiratory duty cycle, or transcutaneous oxygen saturation. However, there was a significant reduction in breathlessness as measured by mean ISO Borg score following salmeterol (4.62 (1.09) $v 3.78$ (1.14); mean difference $-0.84(95 \%$ CI -1.54 to -0.14$)$ ), and change in Borg score from rest to isotime (table 3). In addition, salmeterol led to a significant reduction in the primary outcome measure (ISO IC), accompanied by improvements in operational lung volumes (table 3). Mean (SD) ISO IC was 0.98 (0.44) l following placebo and 1.27 (0.51) l after salmeterol (mean difference 0.29 (95\% CI 0.11 to 0.44 ); $\mathrm{p}=0.002$ ).

\section{Respiratory muscle pressure time products (PTPs)}

Figure 1 shows the effects of salmeterol on ISO PTPs. Salmeterol led to a significant reduction in the primary outcome measure, mean (SD) ISO PTPdi (294.5 (99.6) v 348.6
(95.9) $\mathrm{cm} \mathrm{H}_{2} \mathrm{O} / \mathrm{s} / \mathrm{min}$; mean difference $-54.2 \quad$ (95\% CI -102.5 to -5.9$) ; \mathrm{p}=0.03)$, and also in mean ISO PTPga (544.8 (291.3) v 659.3 (312.2) $\mathrm{cm} \mathrm{H}_{2} \mathrm{O} / \mathrm{s} / \mathrm{min}$; mean difference $-115(95 \%$ CI -225.7 to -3.5$) ; \mathrm{p}=0.04)$.

\section{Improvements in isotime Borg score}

There was no significant linear relationship between improvements in ISO Borg score and changes in respiratory muscle PTP, breathing pattern, minute ventilation, or baseline lung function. However, significant linear correlations were found between changes in ISO $\Delta$ tidal volume/ $\Delta$ oesophageal pressure $(r=0.60 ; \mathrm{p}=0.01), \quad$ EELV/TLC $\%$ $(r=0.58 ; \mathrm{p}=0.02)$ and IC $(r=0.56 ; \mathrm{p}=0.02)$ and ISO Borg score (fig 2 ). On backwards multivariate analysis, only $\Delta$ tidal volume/Aoesophageal pressure and EELV/TLC\% remained significant independent factors.

\section{DISCUSSION}

Using a randomised, placebo controlled, double blind, crossover design, we assessed the effects of a long acting $\beta_{2}$ agonist, salmeterol, on inspiratory and expiratory muscle activity as well as dyspnoea, exercise capacity, and dynamic hyperinflation in patients with poorly reversible COPD. The principal finding is that, in the absence of a spirometric response to a bronchodilator challenge, this treatment can reduce dynamic hyperinflation and respiratory muscle activity in patients with severe COPD, suggesting a physiological basis for a reduction in symptoms.

\section{Significance of findings}

Recent work has emphasised the role of dynamic hyperinflation as a key determinant of exercise induced dyspnoea. ${ }^{67}$ Bronchodilators have also previously been shown to reduce dynamic hyperinflation during exercise in COPD. Belman and colleagues showed that a single dose of albuterol significantly reduced breathlessness and end expiratory/end inspiratory lung volumes during incremental cycle ergometry, ${ }^{6}$ while similar findings were described by O'Donnell and colleagues during endurance cycling following a 3 week period of nebulised ipratropium bromide. ${ }^{7}$ Boni and colleagues have shown a relationship between changes in resting IC following inhaled salbutamol and dyspnoea during light exercise in COPD patients with expiratory flow limitation. ${ }^{21}$

Apart from obvious differences in design, exercise test, and therapeutic drug, our study offers important additional information compared with these previous studies. Our patients were markedly more severe $\left(\mathrm{FEV}_{1} 0.76 \mathrm{l}\right.$ compared with $1.20,1.05$ and $1.75 \mathrm{l}$ ) and none of our patients had any spirometric response to bronchodilator challenge (mean $\mathrm{FEV}_{1}$ change $10 \mathrm{ml}$ compared with 110, 230 and $390 \mathrm{ml}$ ). Despite this, our patients showed comparable improvements in operating lung volumes during exercise with salmeterol.

Table 2 Effect of salmeterol on lung function and respiratory muscle strength

\begin{tabular}{lccll}
\hline & Placebo & Salmeterol & Mean difference $(95 \% \mathrm{Cl})$ & Drug effect $\mathrm{p}$ value \\
\hline $\mathrm{FEV}_{1}$ (I) & $0.74(0.22)$ & $0.78(0.26)$ & $-0.21(-0.40$ to 0.02$)$ & $0.07^{*}$ \\
$\mathrm{VC} \mathrm{(I)}$ & $2.26(0.78)$ & $2.38(0.79)$ & $-0.54(-1.40$ to 0.30$)$ & $0.19^{*}$ \\
$\mathrm{FEV}_{1} / \mathrm{VC}(\%)$ & $35.0(11.5)$ & $33.9(10.1)$ & $-1.1(-3.6$ to 1.3$)$ & 0.34 \\
$\mathrm{TLC}(\mathrm{II})$ & $6.56(1.47)$ & $6.45(1.44)$ & $-0.11(-0.30$ to 0.08$)$ & 0.22 \\
$\mathrm{RV}(\mathrm{I})$ & $4.30(0.82)$ & $4.08(0.90)$ & $-0.23(-0.48$ to 0.03$)$ & 0.08 \\
$\mathrm{RV} / \mathrm{TLC}(\%)$ & $66.2(0.05)$ & $63.6(0.07)$ & $-2.6(-4.91$ to -0.33$)$ & 0.03 \\
PImax & $81.6(14.5)$ & $77.4(12.2)$ & $-4.2(-9.5$ to 1.2$)$ & 0.12 \\
Sniff Pdi & $99.9(10.7)$ & $97.8(16.3)$ & $-2.1(-8.7$ to 4.5$)$ & 0.52 \\
Sniff Poes & $79.6(12.4)$ & $78.8(12.9)$ & $-0.8(-4.7$ to 3.1$)$ & 0.66 \\
\hline
\end{tabular}

Values are mean (SD) or mean treatment differences with $95 \%$ confidence intervals. Respiratory muscle strength tests are measured in $\mathrm{cm} \mathrm{H}_{2} \mathrm{O}$.

$\mathrm{FEV}_{1}=$ forced expiratory volume in 1 second; $\mathrm{VC}=$ vital capacity; $\mathrm{TLC}=$ total lung capacity; $\mathrm{RV}=$ residual volume; PImax = maximum inspiratory pressure; $\mathrm{Pdi}=$ diaphragmatic pressure; $P$ Poes $=$ oesophageal pressure ${ }^{*}$ Significant period $\times$ treatment interaction. 
Table 3 Effect of salmeterol on dyspnoea and operational lung volumes during treadmill exercise

\begin{tabular}{|c|c|c|c|c|}
\hline & Placebo & Salmeterol & Mean difference $(95 \% \mathrm{Cl})$ & p value \\
\hline Rest Borg & $0.0(0.0,0.5)$ & $0.0(0.0,0.5)$ & $0.03(-0.17$ to 0.24$)$ & 0.75 \\
\hline ISO Borg & $4.62(1.09)$ & $3.78(1.14)$ & $-0.84(-1.54$ to -0.14$)$ & 0.02 \\
\hline$\Delta$ Borg & $4.34(1.22)$ & $3.47(1.07)$ & $-0.87(-1.55$ to -0.20$)$ & 0.01 \\
\hline Rest IC (I) & $1.32(0.43)$ & $1.48(0.52)$ & $0.16(0.02$ to 0.32$)$ & $0.03^{*}$ \\
\hline ISO IC (I) & $0.98(0.44)$ & $1.27(0.51)$ & $0.29(0.11$ to 0.44$)$ & 0.002 \\
\hline $\mathrm{DH}(\Delta \mathrm{IC})(\mathrm{I})$ & $0.33(0.20)$ & $0.22(0.15)$ & $-0.11(-0.18$ to -0.04$)$ & 0.002 \\
\hline Rest EELV/TLC (\%) & $80.0(4.0)$ & $76.6(7.7)$ & $-3.4(-6.3$ to -0.6$)$ & 0.02 \\
\hline ISO EELV/TLC (\%) & $85.3(5.1)$ & $80.3(6.9)$ & $-5.0(-8.0$ to -2.2$)$ & 0.002 \\
\hline$\Delta \mathrm{EELV} / \mathrm{TLC}(\%)$ & $5.3(3.3)$ & $3.7(2.6)$ & $-1.6(-2.7$ to -0.7$)$ & 0.003 \\
\hline Rest EILV/TLC (\%) & $90.2(4.2)$ & $87.1(6.0)$ & $-3.1(-5.6$ to -0.6$)$ & 0.02 \\
\hline ISO EILV/TLC (\%) & $98.2(5.7)$ & $93.5(5.3)$ & $-4.7(-7.6$ to -1.8$)$ & 0.004 \\
\hline$\Delta$ EILV/TLC $(\%)$ & $8.0(4.3)$ & $6.4(4.5)$ & $-1.6(-3.3$ to 0.1$)$ & 0.07 \\
\hline ISO $\mathrm{SaO}_{2}(\%)$ & $87.0(6.7)$ & $87.8(6.4)$ & $0.8(-0.4$ to 1.9$)$ & 0.18 \\
\hline Endurance time (s) & $333(151)$ & $347(180)$ & $14(-23.9$ to 52.0$)$ & 0.44 \\
\hline
\end{tabular}

Values are mean (SD) and mean treatment differences with $95 \% \mathrm{Cl}$ except for resting Borg scores where values are median (25th, 75th interquartile range).

Borg = modified Borg dyspnoea scale; $\Delta=$ change from rest to end isotime; $\mathrm{IC}=$ inspiratory capacity; $\mathrm{DH}=$ dynamic hyperinflation; $E E L V=$ end expiratory lung volume; $E I L V=$ end inspiratory lung volume; $T L C=$ total lung capacity; $\mathrm{IRV}=$ inspiratory reserve volume; ISO = isotime

*Significant period $x$ treatment interaction.

O'Donnell and colleagues demonstrated a mean improvement of $390 \mathrm{ml}$ in ISO IC in their patients following acute nebulised ipratropium, similar to the improvement seen in our study. ${ }^{7}$ Similarly, Belman et al reported a mean reduction of $4 \%$ in isotime EELV/TLC\% and a $2 \%$ reduction in ISO EILV/ TLC\% following single dose albuterol, ${ }^{6}$ compared with the $5 \%$ and $4.7 \%$ reduction seen, respectively, in our group. The only other study in patients with COPD of comparable severity ${ }^{22}$ found a mean improvement in resting IC of $210 \mathrm{ml}$ with $5 \mathrm{mg}$ nebulised salbutamol, but the observations were not extended into exercise.

This study is also the first to investigate the effects of bronchodilation on pressure time products during treadmill exercise, demonstrating a reduction in PTPdi and PTPga with salmeterol. For a given neural output, transdiaphragmatic pressure increases as lung volumes fall; the reduction in PTPdi is particularly striking given that operating lung volumes also fell. A previous study has shown a reduction in quiet work of breathing and airway resistance with formoterol, ${ }^{23}$ but these observations were not repeated during exercise. Similarly, Duranti and co-workers ${ }^{24}$ reported a reduction in oesophageal pressure swings, surface diaphragm electromyogram activity, and total lung resistance in patients with severe COPD at rest following a high dose of fenoterol.

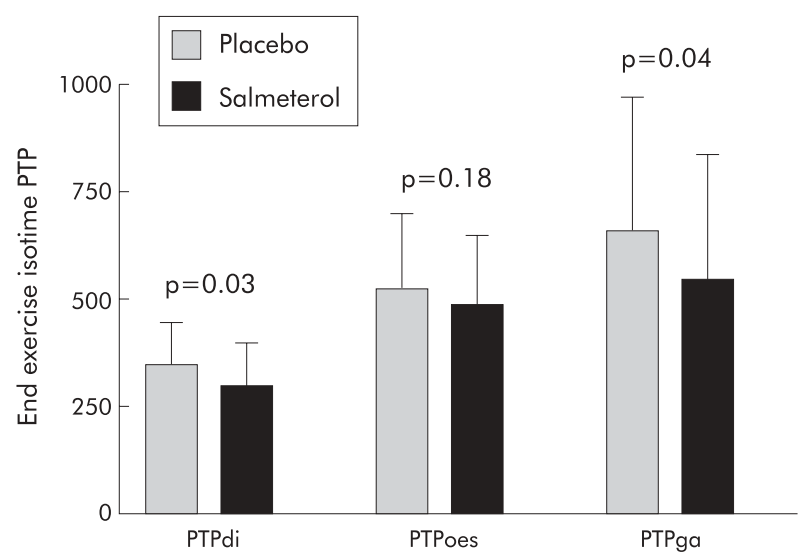

Figure 1 Effect of salmeterol on end exercise isotime respiratory muscle pressure time products.
Dynamic hyperinflation during exercise increases EELV, leading to adverse mechanical sequelae including breathing on a higher and less steep portion of the compliance curve, as well as a positive pressure within the alveoli at the end of expiration. PEEPi acts as an inspiratory threshold load that increases the work of breathing and also places the diaphragm at a mechanical disadvantage. Although PTPdi was reduced at isotime with salmeterol, there was no significant change in PTPoes. This is not altogether surprising, given that hyperinflation is more detrimental to the diaphragm than the rib cage or accessory inspiratory muscles. ${ }^{25}$ The effect on PTPga corroborates previous findings that expiratory muscle activity increases with progressive airflow obstruction and dynamic hyperinflation. ${ }^{26}$ Increased
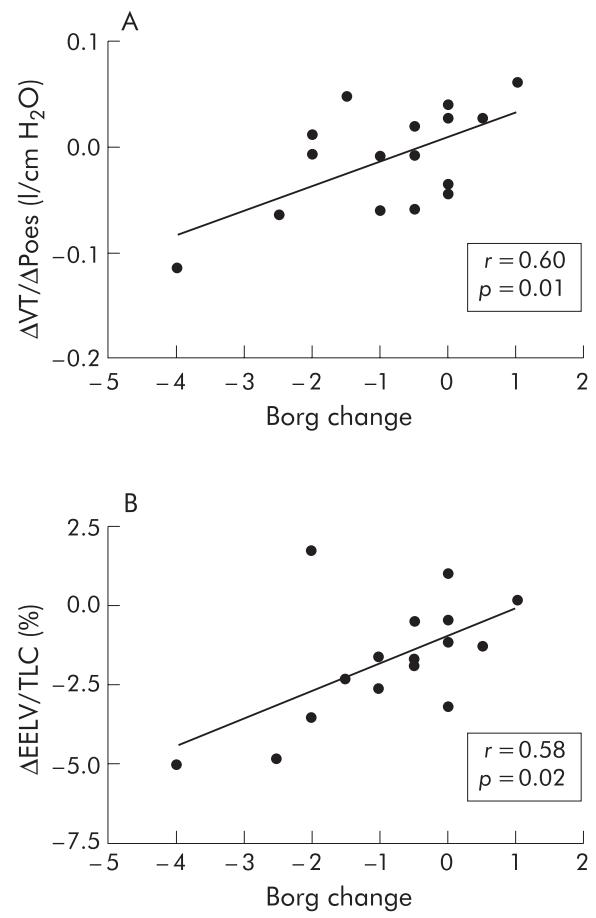

Figure 2 Correlation between improvements in Borg score at isotime with (A) isotime $\Delta$ tidal volume/ $\Delta$ oesophageal pressure and (B) isotime $\triangle \mathrm{EELV} / \mathrm{TLC} \%$. 
expiratory muscle activity cannot contribute to greater expiratory flow, and it remains unclear what role this abdominal muscle activity plays as it should be detrimental in energy terms.

Although salmeterol reduced isotime PTPdi and PTPga, there was no significant linear relationship with improvements in dyspnoea. The best correlates of reduced breathlessness were changes in operational lung volumes, particularly EELV/TLC and IC, and in $\Delta$ tidal volume/ $\Delta$ oesophageal pressure. Current hypotheses on the origin of dyspnoea emphasise the role of neuromechanical dissociation (a disparity between the respiratory drive or motor output and the mechanical response of the system) rather than work of breathing per se. ${ }^{27}$ This is supported by recent work which found a progressive mismatch between diaphragm electromyogram activity and transdiaphragmatic pressure swings during exercise in patients with COPD. ${ }^{28}$ A reduction in dynamic hyperinflation would improve the mechanical response and hence decrease neuromechanical uncoupling.

One potential explanation for our findings could be improved inspiratory muscle contractility with salmeterol as $\beta_{2}$ agonists may increase the maximal force generated by the diaphragm. ${ }^{29}$ However, this is unlikely to play a major role given that no change was seen in the inspiratory muscle strength tests, including sniff Pdi. Alternatively, the improvements in dynamic hyperinflation and operational lung volumes, in the absence of changes in $\mathrm{FEV}_{1}$ or breathing pattern, suggest that salmeterol may exert its effects in the peripheral airways-bronchodilation leading to reduced small airways resistance. ${ }^{23}$

Despite the improvements in exertional dyspnoea, operational lung volumes, and respiratory muscle activity, salmeterol did not improve endurance time. This may partly be due to the relatively small sample size which was designed to demonstrate significant changes in PTPdi rather than changes in exercise capacity. Given the invasive nature of oesophageal and gastric balloon placement on two separate occasions for each patient, it was not appropriate to recruit a larger number of patients. Another possible explanation is quadriceps dysfunction; lower limb muscle weakness is well documented in $\mathrm{COPD}^{30}$ and quadriceps fatigue may contribute to limited exercise tolerance. ${ }^{31}{ }^{32}$ Saey and colleagues have shown that endurance cycling time does not improve following nebulised ipratropium in patients with COPD who develop contractile quadriceps fatigue. ${ }^{31}$ However, it is important to note that quadriceps fatigue is probably a less significant factor during walking. ${ }^{32}$

This study has shown that, despite "non-reversibility" in spirometry, a long acting bronchodilator can lead to physiological improvements in patients with severe COPD. A 2 week treatment period of salmeterol improves exertional dyspnoea and operational lung volumes, and reduces diaphragmatic and abdominal muscle activity. A poor spirometric response to a bronchodilator challenge in COPD patients does not preclude symptomatic and physiological improvements during exercise with a long acting $\beta_{2}$ agonist.

\section{ACKNOWLEDGEMENTS}

The authors thank Tracey Fleming, Claire Wood and the Lung Function Department of King's College Hospital for performing the lung function tests and Toni Maslen and Anne Cheesbrough of GlaxoSmithKline UK for their help in planning the study and for statistical advice.

\section{Authors' affiliations}

W D C Man, N Mustfa, D Nikoletou, S Kaul, G F Rafferty, J Moxham, Respiratory Muscle Laboratory, Guy's, King's and St Thomas' School of Medicine, King's College Hospital, London, UK

N Hart, M I Polkey, Royal Brompton Hospital, London, UK
N Donaldson, Department of Statistics (Research \& Development), King's College Hospital, London, UK

WDCM is a Clinical Research Training Fellow of the Medical Research Council (UK).

This study was funded in part by a project grant from GlaxoSmithKline UK (protocol no SMS40312) who provided study drugs and placebo. They were involved in the initial design of the study but had no input in the writing of the final protocol, patient recruitment, data acquisition or analysis, or in the preparation of the manuscript.

WDCM has received assistance for travel to international conferences from Schering-Plough, GlaxoSmithKline, Allen \& Hanburys. BoehringerIngelheim, and Bayer pharmaceutical companies. SK has received lecture fees from GlaxoSmithKline, Astra Zenca and BoehringerIngelheim. $\mathrm{NH}$ has received assistance for travel to international conferences from Bayer BREAS, 3M, Glaxo Wellcome, Cephalon and MedicAid. MIP has received lecture fees from Astra Zeneca, GlaxoSmithKline, Nutricia and Cephalon, and assistance for travel to international conferences from Glaxo Wellcome, Astra Zeneca, Cephalon, $3 \mathrm{M}$ and Ciba. JM has received assistance for travel to international conferences from Schering-Plough and Pfizer. NM, DN, GFR and ND have no conflicts of interest.

WDCM was involved in the design of the protocol, recruitment of patients, collection and analysis of data, and wrote the first draft of the manuscript. NM, DN, and SK were involved in recruitment of patients, collection of data, and preparation of the final manuscript. NH and GFR were involved in the design of the study, analysis of the data, and preparation of the final manuscript. ND gave statistical support and was involved in the analysis of data and the preparation of the final manuscript. MIP and JM were the senior investigators with the original idea for the study. They designed the study protocol, helped to write the first draft of the manuscript, supervised the conduct of the study and preparation of the final manuscript.

\section{REFERENCES}

1 Pauwels RA, Buist AS, Calverley PM, et al. Global strategy for the diagnosis, management, and prevention of chronic obstructive pulmonary disease. $\mathrm{NHLBI} / \mathrm{WHO}$ Global Initiative for Chronic Obstructive Lung Disease (GOLD) Workshop summary. Am J Respir Crit Care Med 2001; 163:1256-76

2 Jones PW, Bosh TK. Quality of life changes in COPD patients treated with salmeterol. Am J Respir Crit Care Med 1997;155:1283-9.

3 Rennard SI, Anderson W, ZuWallack R, et al. Use of a long-acting inhaled beta2-adrenergic agonist, salmeterol xinafoate, in patients with chronic obstructive pulmonary disease. Am J Respir Crit Care Med 2001;163:1087-92.

4 Calverley P, Pauwels R, Vestbo J, et al. Combined salmeterol and fluticasone in the treatment of chronic obstructive pulmonary disease: a randomised controlled trial. Lancet 2003;361:449-56.

5 Calverley PM, Burge PS, Spencer S, et al. Bronchodilator reversibility testing in chronic obstructive pulmonary disease. Thorax 2003;58:659-64.

6 Belman MJ, Botnick WC, Shin JW. Inhaled bronchodilators reduce dynamic hyperinflation during exercise in patients with chronic obstructive pulmonary disease. Am J Respir Crit Care Med 1996;153:967-75.

7 O'Donnell DE, Lam M, Webb KA. Measurement of symptoms, lung hyperinflation, and endurance during exercise in chronic obstructive pulmonary disease. Am J Respir Crit Care Med 1998;158:1557-65.

8 Field S, Sanci S, Grassino A. Respiratory muscle oxygen consumption estimated by the diaphragm pressure-time index. J Appl Physiol 1984;57:44-51.

9 Kyroussis D, Mills GH, Polkey Ml, et al. Abdominal muscle fatigue after maximal ventilation in humans. J Appl Physiol 1996;81:1477-83.

10 Kyroussis D, Polkey Ml, Hamnegard CH, et al. Respiratory muscle activity in patients with COPD walking to exhaustion with and without pressure support. Eur Respir J 2000;15:649-55.

11 Jubran A, Tobin MJ. Pathophysiologic basis of acute respiratory distress in patients who fail a trial of weaning from mechanical ventilation. Am J Respir Crit Care Med 1997; 155:906-15.

12 COPD Guidelines Group of the Standards of Care Committee of the BTS. BTS guidelines for the management of chronic obstructive pulmonary disease. Thorax 1997;52(Suppl 5):S1-28.

13 Singh SJ, Morgan MD, Scott S, et al. Development of a shuttle walking test of disability in patients with chronic airways obstruction. Thorax 1992;47:1019-24.

14 Yan S, Kaminski D, Sliwinski P. Reliability of inspiratory capacity for estimating end-expiratory lung volume changes during exercise in patients with chronic obstructive pulmonary disease. Am J Respir Crit Care Med 1997; 156:55-9.

15 Borg GA. Psychophysical bases of perceived exertion. Med Sci Sports Exerc 1982;14:377-81 
16 Stubbing DG, Pengelly LD, Morse JL, et al. Pulmonary mechanics during exercise in subjects with chronic airflow obstruction. J Appl Physiol 1980;49:511-5.

17 Marin JM, Carrizo SJ, Gascon M, et al. Inspiratory capacity, dynamic hyperinflation, breathlessness, and exercise performance during the 6-minutewalk test in chronic obstructive pulmonary disease. Am J Respir Crit Care Med 2001;163:1395-9.

18 Miller JM, Moxham J, Green M. The maximal sniff in the assessment of diaphragm function in man. Clin Sci (Lond) 1985:69:91-6.

19 Ninane V, Rypens F, Yernault JC, et al. Abdominal muscle use during breathing in patients with chronic airflow obstruction. Am Rev Respir Dis 1992;146:16-21.

20 Appendini L, Patessio A, Zanaboni S, et al. Physiologic effects of positive end expiratory pressure and mask pressure support during exacerbations of chronic obstructive pulmonary disease. Am J Respir Crit Care Med 1994; 149:1069-76.

21 Boni E, Corda L, Franchini D, et al. Volume effect and exertional dyspnoea after bronchodilator in patients with COPD with and without expiratory flow limitation at rest. Thorax 2002;57:528-32.

22 Hadcroft J, Calverley PM. Alternative methods for assessing bronchodilator reversibility in chronic obstructive pulmonary disease. Thorax 2001;56:713-20.

23 Maesen BL, Westermann CJ, Duurkens VA, et al. Effects of formoterol in apparently poorly reversible chronic obstructive pulmonary disease. Eur Respir J 1999;13:1103-8.
24 Duranti R, Misuri G, Gorini M, et al. Mechanical loading and control of breathing in patients with severe chronic obstructive pulmonary disease. Thorax 1995;50:127-33.

25 Decramer M. Hyperinflation and respiratory muscle interaction. Eur Respir J 1997; 10:934-41.

26 Ninane V, Yernault JC, de Troyer A. Intrinsic PEEP in patients with chronic obstructive pulmonary disease. Role of expiratory muscles. Am Rev Respir Dis 1993; 148:1037-42.

27 Manning HL, Schwartzstein RM. Pathophysiology of dyspnea. N Engl J Med 1995;333:1547-53.

28 Sinderby C, Spahija J, Beck J, ef al. Diaphragm activation during exercise in chronic obstructive pulmonary disease. Am J Respir Crit Care Med 2001; 163:1637-41.

29 Aubier M, Viires N, Murciano D, et al. Effects and mechanism of action of terbutaline on diaphragmatic contractility and fatigue. J Appl Physiol 1984;56:922-9.

30 Man WD, Soliman MG, Nikoletou D, et al. Non-volitional assessment of skeletal muscle strength in patients with chronic obstructive pulmonary disease. Thorax 2003:58:665-9.

31 Saey D, Debigare R, LeBlanc $P$, et al. Contractile leg fatigue after cycle exercise: a factor limiting exercise in patients with chronic obstructive pulmonary disease. Am J Respir Crit Care Med 2003;168:425-30.

32 Man WD, Soliman MG, Gearing J, et al. Symptoms and quadriceps fatigability after walking and cycling in chronic obstructive pulmonary disease. Am J Respir Crit Care Med 2003;168:562-7.

\section{LUNG ALERT}

Budesonide/formoterol may be used for adjustable maintenance dosing in asthma $\Delta$ Aalbers R, Backer V, Kava TKK, et al. Adjustable maintenance dosing with budesonide/formoterol compared with fixeddose salmeterol/fluticasone in moderate to severe asthma. Curr Med Res Opin 2004;20:225-40

$\mathrm{T}$ his randomised, double blind/open two phase multicentre study, sponsored by Astra Zeneca, was conducted over 7 months to find out if asthma control was better when patients adjusted the maintenance dose of inhaled budesonide/formoterol (Symbicort Turbohaler) according to asthma symptoms than when fixed dose (FD) regimens were used.

Eligible patients were randomised to one of three groups: (1) Symbicort FD 160/4.5 $\mu \mathrm{g}$, two inhalations twice daily (bd); (2) Symbicort adjustable maintenance dose (AMD) 160/ $4.5 \mu \mathrm{g}$, two inhalations bd; or (3) salmeterol/fluticasone FD (Seretide Diskus) 50/250 $\mu \mathrm{g}$, one inhalation bd. The study consisted of three treatment periods: run in (10-14 days), double blind ( 1 month with either Symbicort FD or Seretide FD), and open extension (6 months). In the open extension phase, those in the Symbicort AMD arm were advised to reduce the number to one inhalation bd or to increase the number of inhalations to four bd for at least 7 days according to the frequency of reliever usage and nocturnal awakenings due to asthma.

Symbicort FD significantly increased $\mathrm{FEV}_{1}$ compared with Seretide FD $(\mathrm{p}<0.05)$. Comparable improvements in FEV 1 were observed with Symbicort AMD and Seretide FD. Patients in the Symbicort AMD group had significantly fewer exacerbations/hospital attendances/oral steroid courses $(p<0.018)$ and lower use of reliever medication $(p<0.01)$ than those in the Seretide FD group. There was no difference in the number of "well controlled asthma weeks" (WCAW) between the two FD groups, but Symbicort AMD increased the odds of achieving a WCAW compared with Symbicort FD $(\mathrm{p}=0.049)$.

The results suggest that FD regimens with Symbicort and Seretide provide similar levels of asthma control. Total drug usage was lower with Symbicort AMD than FD. This study reinforces the need for home management plans and empowerment of patients to manage their asthma at home.

D Simcock

Specialist Registrar, Respiratory Medicine, Newham General Hospital, London, UK; dsimcock@doctors.org.uk 